Résumés des conférences et travaux

\title{
Historiographie de la Renaissance dans le domaine de l'architecture : méthodes et approches
}

Conférences de l'année 2012-2013

\section{Antonio Brucculeri}

\section{OpenEdition \\ Journals}

Electronic version

URL: https://journals.openedition.org/ashp/1619

DOI: 10.4000/ashp.1619

ISSN: 1969-6310

\section{Publisher}

Publications de l'École Pratique des Hautes Études

\section{Printed version}

Date of publication: 1 September 2014

Number of pages: $245-246$

ISSN: 0766-0677

\section{Electronic reference}

Antonio Brucculeri, "Historiographie de la Renaissance dans le domaine

de l'architecture : méthodes et approches", Annuaire de l'École pratique des hautes études (EPHE),

Section des sciences historiques et philologiques [Online], 145 | 2014, Online since 15 December 2014,

connection on 06 July 2021. URL: http://journals.openedition.org/ashp/1619 ; DOI: https://doi.org/

10.4000/ashp.1619 


\title{
HISTORIOGRAPHIE DE LA RENAISSANCE DANS LE DOMAINE DE L'ARCHITECTURE MÉTHODES ET APPROCHES
}

\author{
Chargé de conférences : M. Antonio Brucculeri
}

Programme de l'année 2012-2013 : Le XIXe siècle et la Renaissance (suite).

Dans le cadre du cycle triennal de conférences démarré depuis l'année universitaire 2011-2012, nous avons choisi d'analyser les réceptions de l'architecture de la Renaissance italienne au XIX ${ }^{\mathrm{e}}$ siècle. Nous avons saisi trois axes de réflexion : 1) l'évolution des outils d'étude des bâtiments de la Renaissance, depuis le relevé et le redessin, jusqu'à la photographie et au dessin original, ce qui a fait l'objet de notre cycle de conférences en 2012 ; 2) les définitions de l'idée de Renaissance élaborées non seulement dans le domaine de l'historiographie artistique mais aussi par d'autres formes d'expression littéraire, axe auquel nous avons consacré les conférences de cette année universitaire 2012-2013; 3) pour conclure, l'attention portée aux édifices de la période de la Renaissance en tant que répertoire de références et de modèles pour l'architecture bâtie au XIX ${ }^{\mathrm{e}}$ siècle, sujet auquel une troisième série de conférences sera consacrée en 2014.

Pendant cette année 2012-2013, nous avons abordé la construction et la diffusion du concept de Renaissance, à travers des développements sur les différentes formes littéraires concernées. Il a été question d'explorer le domaine des écrits d'histoire générale tout d'abord ; mais aussi le terrain des ouvrages d'esthétique et de théorie de l'art, celui des ouvrages de théorie architecturale, voire le genre littéraire du roman. Il a d'ailleurs été question de multiplier les enquêtes dans de différents contextes nationaux, en comparant en particulier les milieux allemand, britannique et français, et leurs évolutions. C'est par ce cadre articulé que nous avons restitué les multiples images de la Renaissance italienne diffusées dès le dernier quart du XVIII ${ }^{\mathrm{e}}$ siècle et jusqu'aux années 1870 . Et c'est à l'intérieur de ce cadre que nous avons resitué les premiers essais d'historiographie artistique consacrés à l'architecture de la Renaissance.

Après une séance introductive illustrant les principaux thèmes et les enjeux méthodologiques de nos conférences, nous avons orienté le regard sur les figures littéraires de la Renaissance au XIX ${ }^{\mathrm{e}}$ siècle à partir de plusieurs observatoires. Avant d'aborder la place que l'architecture de la Renaissance occupe dans le projet éditorial de l'Histoire de l'art par les monumens (1810-1823) de Jean-Baptiste Séroux d'Agincourt, nous avons souligné l'essor d'une nouvelle approche de la période de la Renaissance au courant de la seconde moitié du XVIII ${ }^{\mathrm{e}}$ siècle, à la fois dans le cadre de la spéculation philosophique (Voltaire) et de l'érudition historique (Gibbon). Nous sommes ainsi parvenus à l'examen des monographies que William Roscoe consacre à Laurent le Magnifique et à Léon X, respectivement en 1795 et en 1805. Dans ce contexte, nous avons replacé la genèse de la catégorie même de Renaissance dans le milieu 
francophone, à la fois par les débuts de l'historiographie artistique (Séroux d'Agincourt) mais aussi à travers l'histoire générale, que nous avons interrogée en questionnant la notion de Renaissance en tant que catégorie historiographique, mobilisée par Jules Michelet dans le tome VII de son Histoire de France (1855). Nous avons donc remis en perspective, par rapport à ce cadre plus large, l'apport du milieu germanophone et, en particulier, la manière par laquelle Jacob Burckhardt a abordé la notion de Renaissance dans le domaine de l'histoire de l'art, non seulement dans son célèbre ouvrage sur la civilisation de la Renaissance en Italie (1860) mais aussi dans sa contribution majeure à propos d'histoire architecturale, Geschichte der neueren Baukunst : die Renaissance in Italien (1867). Cela nous a permis de mettre l'accent sur les croisements, chez Burckhardt, entre histoire culturelle, histoire de l'art et histoire de l'architecture, à la fois dans sa pensée et dans son écriture d'historien. L'approche épistémologique qui se dégage des écrits de Burckhardt a été par ailleurs comparée à l'approche d'un architecte et théoricien de l'architecture tel que Gottfried Semper, qui s'intéresse à la Renaissance architecturale en Italie par un point de vue opérationnel, en exploitant les modèles et leurs syntaxes dans ses propres projets d'architecture. Nous avons ensuite brossé un tableau de l'emploi et de la diffusion de la catégorie de Renaissance dans le cadre culturel de l'Angleterre victorienne, en plongeant dans la variété des expériences littéraires. Les dernières conférences ont été conçues autour de deux cas d'études : d'une part les publications d'un essayiste tel que John Addington Symonds, l'auteur de The Renaissance. An Essay (1863) et de Renaissance in Italy (1875-1881); d'autre part les narrations d'une romancière tel que George Eliot, dont nous avons spécialement considéré la construction littéraire du roman Romola (1863). Alors que le premier cas d'étude a été l'occasion pour une plus large mise en perspective entre critique d'art (John Ruskin), théorie architecturale (Augustus Welby Pugin) et - plus loin - approche historiographique (Walter Pater), par le second nous avons enquêté sur la mise en scène de la Renaissance par la fiction historique-littéraire. Audelà de la représentation du milieu florentin à l'époque de Savonarola fournie par l'autrice, l'analyse textuelle du roman Romola nous a permis de porter le regard sur des questionnements plus généraux quant à l'apport des écrivains dans la fabrication du mythe de la Renaissance et dans la représentation même de la Renaissance à travers la description littéraire des villes et des édifices de l'époque.

Les conférences ont été suivies par un nombre restreint d'étudiants et d'auditeurs. Les uns et les autres ont pourtant manifesté un intérêt constant tout au long de la période pendant laquelle les conférences se sont déroulées. Les nombreuses occasions d'échanges et de débat sur les sujets abordés ont confirmé cet intérêt, aussi en liaison avec les discussions au sein du séminaire international L'architecture de la Renaissance au XIX $X^{e}$ siècle que nous avons parallèlement organisé, avec $\mathrm{M}^{\mathrm{me}}$ Sabine Frommel, entre février et juin 2013. 